\title{
Soft-Matrices Computation for Feature Selection on Traditional Malay Musical Instrument Sounds Dataset
}

\author{
Sarjon Defit Tutut Herawan \\ Universitas Putra Indonesia "YPTK" Padang \\ Jl. Raya Lubuk Begalung Padang, Sumatera Barat, Indonesia \\ sarjond@yahoo.co.uk \\ ${ }^{2}$ Ahmad Dahlan University, Yogyakarta, Indonesia \\ AMCS Research Center, Yogyakarta, Indonesia \\ tutut@uad.ac.id
}

\begin{abstract}
Soft set theory proposed by Molodtsov is a new mathematical tool for dealing with the uncertain data. Soft set-based dimensionality reduction can be considered as a technique for feature selection. In this paper, we present an alternative technique for feature selection of traditional Malay musical instrument sounds dataset. The technique is based on matrices computation of multi-soft sets for dimensionality reduction. The dataset is taken from our previous work which consists of 1116 instances and 37 attributes of Malay Musical Instrument Sounds Dataset. The result shows that the obtained features of proposed technique are 35 out of 37 features.
\end{abstract}

Keywords: Soft set theory, Feature selection, Traditional Malay musical instruments sound dataset

\section{Introduction}

In making sure data made available for the process of decision making is sufficient enough, then reduction of data ought to be properly addressed. The main objective of reduction is to lessen the number of parameters, and at the same time, attaining the property of information in helping the process of decision making. The theory of soft set [1] proposed by Molodtsov 1999 is a new method for handling uncertain data. Soft sets are called elementary neighborhood systems. Molodtsov pointed out that one of the main advantages of soft set theory is that it is free from the inadequacy of the parameterization tools, like in the theories of fuzzy set, probability and interval mathematics [1]. As for standard soft set, it may be redefined as the classification of objects in two distinct classes (yes/1 and no/0), thus confirming that soft set can deal with a Boolean-valued information system. One of the potential applications of soft set theory is to be used as the dimensionality reduction and decision making method [2-15]. However, most of the current applications are deal with the Boolean information system [2-7, 12, 13, 15]. To date, less effort has been done in handling the non-Boolean dataset especially for the categorical dataset. In previous papers, Herawan and Mustafa proposed the idea of multisoft sets for representing a categorical-valued information system [16]. Sub sequentially, Herawan, et al., proposed the concept of matrices representation of multi-soft sets and employ such matrices for finding reducts in a categorical-valued information system [17].

In this paper, we employ our early works in [17] as a feature selection technique. It is based on matrices computation of multi-soft sets and further applied it in handling Traditional Malay musical instruments sounds dataset. To accomplish this study, the dataset consists of 1116 instances with 37 attributes from two categories of features schemes which are perception-based and MFCC is exploited. In order to utilize the soft set theory, it is essential to transform the original dataset (continuous values with non- 
categorical form) into discretized values with categorical form. For that, equal width and equal frequency binning algorithm in [18] is employed as the discretization method. Afterwards, the matrices computation of multi-soft sets method for soft dimensionality reduction in multi-valued information system in [17] is adopted to select the best feature set from the large number of features available.

The rest of this paper is organized as follows: Section 2 discuss the theory of soft set and multi-soft sets will be explained in Section 3. Section 4 describes the modeling process of this study. A discussion of the result can be found in Section 5 followed by the conclusion in Section 6.

\section{Soft Set Theory}

Presently, research on soft sets has been very active and many important results have been achieved in the theoretical aspect.

\subsection{Information System}

Data are often presented as a table, columns of which are labeled by attributes, rows by objects of interest and entries of the table are attribute values. By an information system, we mean a 4-tuple (quadruple) $S=(U, A, V, f)$, where $U$ is a non-empty finite set of objects, $A$ is a non-empty finite set of attributes, $V=\bigcup_{a \in A} V_{a}, V_{a}$ is the domain (value set) of attribute $a, f: U \times A \rightarrow V$ is a total function such that $f(u, a) \in V_{a}$, for every $(u, a) \in U \times A$, called information (knowledge) function. An information system is also called a knowledge representation systems or an attribute-valued system and can be intuitively expressed in terms of an information table (refer to Table 1). In many applications, there is an outcome of classification that is known. This a posteriori knowledge is expressed by one (or more) distinguished attribute called decision attribute; the process is known as supervised learning. An information system of this kind is called a decision system, $S=(U, A \bigcup\{d\}, V, f)$.

Definition 1. Let $S=\left(U, A, V_{\{0,1\}}, f\right)$ Abe an information system. If $V_{a}=\{0,1\}$, for every $a \in A$, then $S=\left(U, A, V_{\{0,1\}}, f\right)$ is called a Boolean-valued information system.

\section{Table 1. An Information System}

\begin{tabular}{|c|c|c|c|c|c|c|}
\hline U/A & $a_{1}$ & $a_{2}$ & $\cdots$ & $a_{k}$ & $\cdots$ & \multicolumn{2}{|c|}{$a_{|A|}$} \\
\hline$u_{1}$ & $f\left(u_{1}, a_{1}\right)$ & $f\left(u_{1}, a_{2}\right)$ & $\cdots$ & $f\left(u_{1}, a_{k}\right)$ & $\cdots$ & $f\left(u_{1}, a_{|A|}\right)$ \\
\hline$u_{2}$ & $f\left(u_{2}, a_{1}\right)$ & $f\left(u_{2}, a_{2}\right)$ & $\cdots$ & $f\left(u_{2}, a_{k}\right)$ & $\cdots$ & $f\left(u_{2}, a_{|A|}\right)$ \\
\hline$u_{3}$ & $f\left(u_{3}, a_{1}\right)$ & $f\left(u_{3}, a_{2}\right)$ & $\cdots$ & $f\left(u_{3}, a_{k}\right)$ & $\cdots$ & $f\left(u_{3}, a_{|A|}\right)$ \\
\hline$\vdots$ & $\vdots$ & $\vdots$ & $\ddots$ & $\vdots$ & $\ddots$ & $\vdots$ \\
\hline$u_{|U|}$ & $f\left(u_{|U|}, a_{1}\right)$ & $f\left(u_{|U|}, a_{2}\right)$ & $\cdots$ & $f\left(u_{|U|}, a_{k}\right)$ & $\cdots$ & $f\left(u_{|U|}, a_{|A|}\right)$ \\
\hline
\end{tabular}

\subsection{Soft Set}

Throughout this section $U$ refers to an initial universe, $E$ is a set of parameters in relation to objects in $U, P(U)$ is the power set of $U$. 
Definition 2. (See [1].) A pair $(F, E)$ is called a soft set over $U$, where $F$ is a mapping given by $F: E \rightarrow P(U)$.

In other words, a soft set over $U$ is a parameterized family of subsets of the universe $U$. For $e \in E, F(e)$ may be considered as a set of $e$-elements of the soft set $(F, E)$ or as the set of $e$-approximate elements of the soft set. Clearly, a soft set is not a (crisp) set. Based on the definition of an information system and a soft set, in this section we show that a soft set is a special type of information systems, i.e., a Boolean-valued information system.

Example 1. Based on [13], let consider a soft set $(F, E)$ which describes the "conditions of patients suspected influenza" that a hospital is considering to make a decision. The six influenza symptoms, i.e., fever, respiratory, nasal discharges, cough, headache and lethargic. Suppose that there are six patients in the hospital under consideration, $U=\left\{p_{1}, p_{2}, p_{3}, p_{4}, p_{5}, p_{6}\right\}, \quad$ and $E$ is a set of decision parameters $E=\left\{e_{1}, e_{2}, e_{3}, e_{4}, e_{5}, e_{6}, e_{7}\right\}$, where is the parameter "fever", is the parameter "respiratory", is the parameter "nasal discharges", is the parameter "cough", is the parameter "headache", is the parameter "sore throat", is the parameter "lethargic"

Consider the mapping $F: E \rightarrow P(U)$ given by "patients $(\cdot)$ ", where $($.$) is to be filled in$ by one of parameters $e \in E$. Suppose that $F\left(e_{1}\right)=\left\{p_{1}, p_{3}, p_{4}, p_{5}, p_{6}\right\}$, $F\left(e_{2}\right)=\left\{p_{1}, p_{2}\right\}, \quad F\left(e_{3}\right)=\left\{p_{1}, p_{2}, p_{4}\right\}, \quad F\left(e_{4}\right)=\left\{p_{1}\right\}, \quad F\left(e_{5}\right)=\left\{p_{3}, p_{4}\right\}$, $F\left(e_{6}\right)=\left\{p_{2}, p_{4}\right\}$, and $F\left(e_{7}\right)=\left\{p_{1}, p_{3}, p_{5}, p_{6}\right\}$. Therefore, $F\left(e_{1}\right)$ means "patients suffer fever", whose functional value is the set $\left\{p_{1}, p_{3}, p_{4}, p_{5}, p_{6}\right\}$. Thus, we can view the soft set $(F, E)$ as a collection of approximations as below:

$$
(F, E)=\left\{\begin{array}{c}
\text { fever }=\left\{p_{1}, p_{3}, p_{4}, p_{5}, p_{6}\right\}, \\
\text { respiratory }=\left\{p_{1}, p_{2}\right\}, \\
\text { nasal discharges }=\left\{p_{1}, p_{2}, p_{4}\right\}, \\
\text { cough }=\left\{p_{1}\right\}, \\
\text { head ache }=\left\{p_{3}, p_{4}\right\}, \\
\text { sore throat }=\left\{p_{2}, p_{4}\right\}, \\
\text { lethargic }=\left\{p_{1}, p_{3}, p_{5}, p_{6}\right\}
\end{array}\right\}
$$

The relation between a soft set and a Boolean-valued information system is given in the following proposition.

Proposition 1. If $(F, A)$ is a soft set over the universe $U$, then $(F, A)$ is a Booleanvalued information system $S=\left(U, A, V_{\{0,1\}}, f\right)$.

Proof. Let $(F, A)$ be a soft set over the universe $U$, we define a mapping $F=\left\{f_{1}, f_{2}, \cdots, f_{n}\right\}$, where $f_{i}: U \rightarrow V_{i}$ and $f_{i}(x)=\left\{\begin{array}{ll}1, & x \in F\left(a_{i}\right) \\ 0, & x \notin F\left(a_{i}\right)\end{array}\right.$, for $1 \leq i \leq|A|$.

Hence, if $V=\bigcup_{a_{i} \in A} V_{a_{i}}$, where $V_{e_{i}}=\{0,1\}$, then a soft set $(F, A)$ can be considered as a Boolean-valued information system $S=\left(U, A, V_{\{0,1\}}, f\right)$. 
From Proposition 1, it is easily to understand that a Boolean-valued information system can be represented as a soft set. Thus, we can make a one-to-one correspondence between $(F, E)$ over $U$ and $S=\left(U, A, V_{\{0,1\}}, f\right)$. The tabular representation of the above soft set is given in the Table 2. From Table 2, each approximation has two parts, a predicate $e$ and an approximate value set $p$. For example, for the approximation " fever $=\left\{p_{1}, p_{3}, p_{4}, p_{5}, p_{6}\right\}$ ", we have the predicate name of patients with fever and its value set is $\left\{p_{1}, p_{3}, p_{4}, p_{5}, p_{6}\right\}$.

\section{Table 2. Conditions of Patients Suspected Influenza}

\begin{tabular}{|c|c|c|c|c|c|c|c|}
\hline$U / E$ & $e_{1}$ & $e_{2}$ & $e_{3}$ & $e_{4}$ & $e_{5}$ & $e_{6}$ & $e_{7}$ \\
\hline$p_{1}$ & 1 & 1 & 1 & 1 & 0 & 0 & 1 \\
\hline$p_{2}$ & 0 & 1 & 1 & 0 & 0 & 1 & 0 \\
\hline$p_{3}$ & 1 & 0 & 0 & 0 & 1 & 0 & 1 \\
\hline$p_{4}$ & 1 & 0 & 1 & 0 & 1 & 1 & 0 \\
\hline$p_{5}$ & 1 & 0 & 0 & 0 & 0 & 0 & 1 \\
\hline$p_{6}$ & 1 & 0 & 0 & 0 & 0 & 0 & 1 \\
\hline
\end{tabular}

Definition 3. (See [19].) The class of all value sets of a soft set $(F, E)$ is called valueclass of the soft set and is denoted by $C_{(F, E)}$.

\section{Method and Main Result}

The "standard" soft set deals with a Boolean-valued information system. For a multivalued information system $S=(U, A, V, f)$, where $V=\bigcup_{a \in A} V_{a}, V_{a}$ is the domain (value set) of attribute $a$ which has multi value, a decomposition can be made from $S$ into $|A|$ number of Boolean-valued information systems $S=\left(U, A, V_{\{0,1\}}, f\right)$. In this subsection, we present the construction of multi-soft sets representing $S=(U, A, V, f)$ based on such decomposition [16].

\subsection{Multi-soft Sets}

In the following sub-section, we propose an idea of decomposition of a multi-valued information system $S=(U, A, V, f)$ into $|A|$-numbers of Boolean-valued information system $S^{i}=\left(U, a_{i}, V_{\{0,1\}}, f\right)$, where $|A|$ is the cardinality of $A$.

\subsection{Decomposition of a Multi-valued Information System}

The decomposition of $S=(U, A, V, f)$ is based on decomposition of $A=\left\{a_{1}, a_{2}, \cdots, a_{|A|}\right\}$ into the disjoint-singleton attribute $\left\{a_{1}\right\},\left\{a_{2}\right\}, \cdots,\left\{a_{|A|}\right\}$. Here, we only consider for complete information systems. Let $S=(U, A, V, f)$ be an information system such that for every $a \in A, V_{a}=f(U, A)$ is a finite non-empty set and for every $u \in U,|f(u, a)|=1$. For every $a_{i}$ under $i^{\text {th }}$-attribute consideration, $a_{i} \in A$ and $v \in V_{a}$, we define the map $a_{v}^{i}: U \rightarrow\{0,1\}$ such that $a_{v}^{i}(u)=1$ if $f(u, a)=v$, otherwise 
$a_{v}^{i}(u)=0$. The next result, we define a Boolean-valued information system as a quadruple $S^{i}=\left(U, a_{i}, V_{\{0,1\}}, f\right)$. The information systems $S^{i}=\left(U, a_{i}, V_{\{0,1\}}, f\right)$, $1 \leq i \leq|A|$ is referred to as a decomposition of a multi-valued information system $S=(U, A, V, f)$ into $|A|$ Boolean-valued information systems, as depicted in Figure 1.

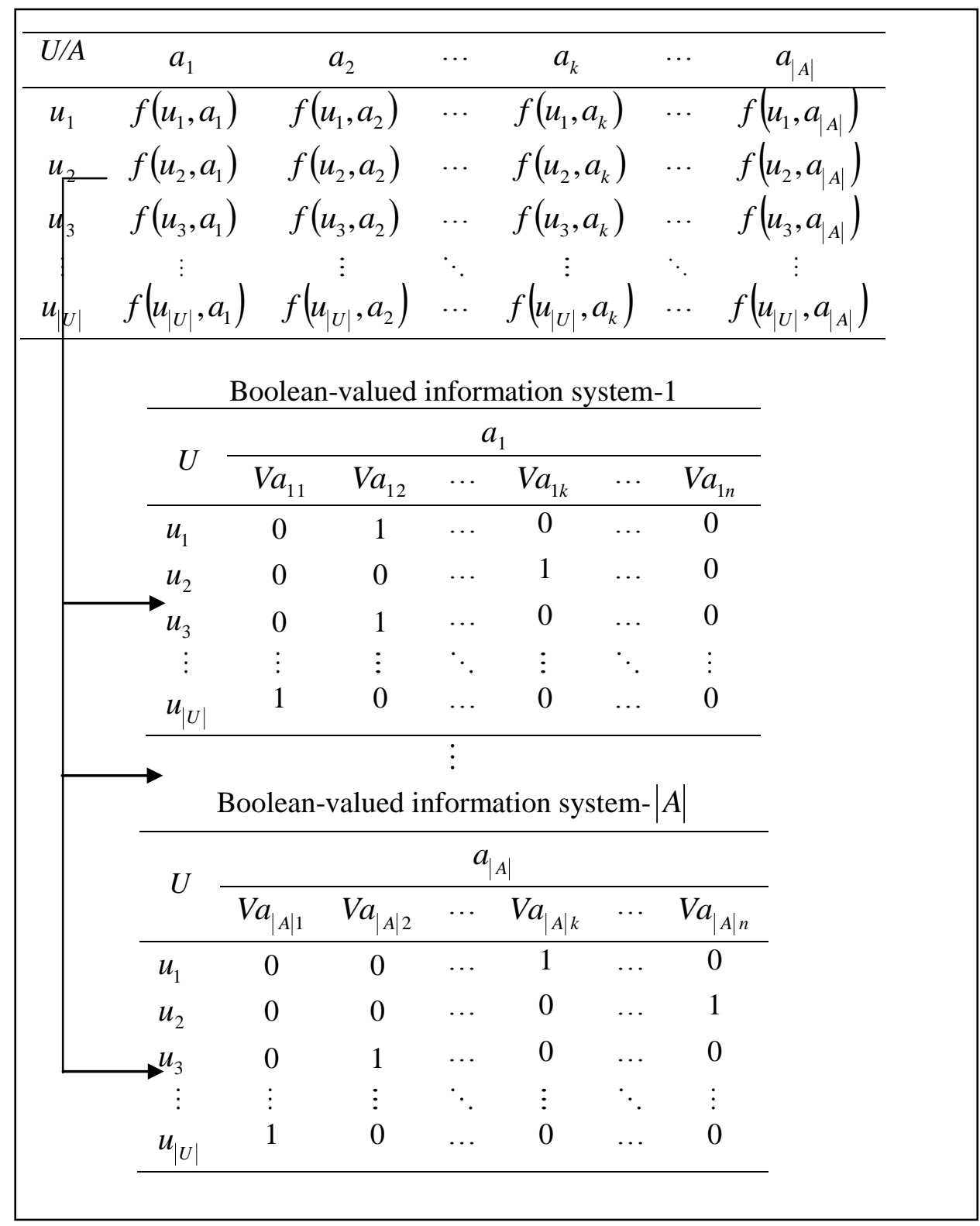

Figure 1. A Decomposition of Information Systems

Every information system $S^{i}=\left(U, a_{i}, V_{a i}, f\right), \quad 1 \leq i \leq|A|$ is a deterministic information system since for every $a \in A$ and for every $u \in U,|f(u, a)|=1$ such that the structure of a multi-valued information system and $|A|$ number of Boolean-valued information systems give the same value of attribute related to objects. 


\subsection{Multi-soft Sets Construction}

The idea of multi-soft sets is based on a decomposition of a multi-valued information system $S=(U, A, V, f)$, into $|A|$ number of Boolean-valued information systems $S=\left(U, A, V_{\{0,1\}}, f\right)$, where $|A|$ denotes the cardinality of $A$. Consequently, the $|A|$ Boolean-valued information systems define multi-soft sets $(F, A)=\left\{\left(F, a_{i}\right): 1 \leq i \leq|A|\right\}$.

Based on the notion of a decomposition of a multi-valued information system in the previous section, in this sub-section we present the notion of multi-soft set representing multi-valued information systems. Let $S=(U, A, V, f)$ be a multi-valued information system and $S^{i}=\left(U, a_{i}, V_{a i}, f\right), 1 \leq i \leq|A|$ be the $|A|$ Boolean-valued information systems. From Proposition 1, we have

$$
S=(U, A, V, f)=\left\{\begin{array}{cc}
S^{1}=\left(U, a_{1}, V_{\{0,1\}}, f\right) & \Leftrightarrow\left(F, a_{1}\right) \\
S^{2}=\left(U, a_{2}, V_{\{0,1\}}, f\right) & \Leftrightarrow\left(F, a_{2}\right) \\
\vdots & \vdots \quad \vdots \\
S^{|A|}=\left(U, a_{|A|}, V_{\{0,1\}}, f\right) & \Leftrightarrow\left(F, a_{|A|}\right) \\
& =\left(\left(F, a_{1}\right),\left(F, a_{2}\right), \cdots,\left(F, a_{|A|}\right)\right)
\end{array}\right.
$$

We define $(F, A)=\left(\left(F, a_{1}\right),\left(F, a_{2}\right), \cdots,\left(F, a_{|A|}\right)\right)$ as a multi-soft sets over universe $U$ representing a multi-valued information system $S=(U, A, V, f)$.

\subsection{Matrices Representation of Multi-Soft Sets}

The concept of matrix representing multi-soft sets is described here. In this sub-section, the notation $(F, A)$ represents a multi-soft set over universe $U$ of a multi-valued information system $S=(U, A, V, f)$.

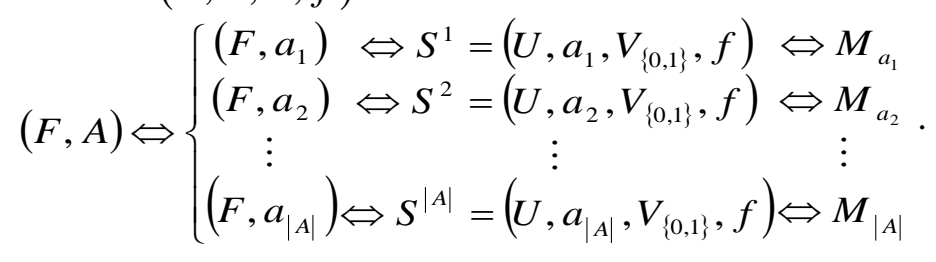

Definition 4. Matrix $M_{a_{i}}, 1 \leq i \leq|A|$ is called matrix representation of soft set $\left(F, a_{1}\right)$ over universe $U$. The dimension of matrices is defined by $\operatorname{dim}\left(M_{a_{i}}\right)=|U| \times\left|V a_{i}\right|$. All entries of $M_{a_{i}}=\left[a_{i j}\right]$ is belong to a set $\{0,1\}$, where

$$
a_{i j}=\left\{\begin{array}{lll}
0, & \text { if } & |f(u, \alpha)|=0,1 \leq i \leq|U|, 1 \leq j \leq\left|V a_{i}\right|, u \in U \text { and } \alpha \in V a_{i} . \\
1, & \text { if } \quad|f(u, \alpha)|=1
\end{array}\right.
$$

The collection of all matrices representing $(F, A)$ is denoted by $\mathcal{M}_{A}$, i.e.,

$$
\mathcal{M}_{A}=\left\{M_{a_{i}}: 1 \leq i \leq|A|\right\} \text {. }
$$

Definition 5. Let $M_{a_{i}} \in \mathcal{M}_{A}$ be a matrix representation of a soft set $\left(F, a_{i}\right)$ over $U$. The value-class of $M_{a_{i}}$, i.e., class of all value sets of $M_{a_{i}}$, denoted $C_{M_{a_{i}}}$ is defined by

$$
C_{M_{a_{i}}}=\left\{\left\{u:\left|f\left(u, \alpha_{1}\right)\right|=1\right\}, \cdots,\left\{u: \mid f\left(u, \alpha_{\left|V a_{i}\right|}\right)=1\right\}\right\},
$$


where $1 \leq i \leq\left|V a_{i}\right|, u \in U$ and $\alpha \in V a_{i}$.

Clearly $C_{M_{a_{i}}} \subseteq P(U)$.

\subsection{AND and OR Operations in a Collection of Matrices}

The AND and OR operation of the matrices are described in this sub-section.

Definition 6. Let $M_{a_{i}}=\left[a_{k l}\right], 1 \leq k \leq|U|$, for $1 \leq l \leq\left|V_{a_{i}}\right|$ and $M_{a_{j}}=\left[a_{m n}\right]$, for $1 \leq m \leq|U|, \quad 1 \leq n \leq\left|V_{a_{i}}\right|$ be two matrices in $\mathcal{M}_{A}$. The "AND" operation $M_{a_{i}} \mathrm{ANDM}_{a_{j}}$ of matrices $M_{a_{i}}$ and $M_{a_{j}}$ is defined as follows

$$
M_{a_{i}} \mathrm{ANDM}_{a_{j}}=M_{a_{i j}}=\left[a_{p q}\right] \text { with } \operatorname{dim}\left(M_{a_{i j}}\right)=|U| \times\left(\left|V a_{i}\right| \times\left|V a_{j}\right|\right),
$$

where

$$
\left.a_{p 1}=\min \left\{a_{k 1}, a_{m 1}\right\}, a_{p 2}=\min \left\{a_{k 1}, a_{m 2}\right\}, \ldots, a_{p\left(V a_{i}|\times| V a_{j} \mid\right.}\right)=\min \left\{a_{k\left|V a_{i}\right|}, a_{m\left|V a_{j}\right|}\right\} .
$$

Definition 6. Let $M_{a_{i}}=\left[a_{k l}\right]$ and $M_{a_{j}}=\left[b_{m n}\right]$ be two matrices in $\mathcal{M}_{A}$. The "OR" operation $M_{a_{i}} \mathrm{OR} M_{a_{j}}$ of matrices $M_{a_{i}}$ and $M_{a_{j}}$ is defined as follows

$$
M_{a_{i}} \mathrm{OR} M_{a_{j}}=M_{a_{i j}}=\left[c_{p q}\right] \text { with } \operatorname{dim}\left(M_{a_{i j}}\right)=|U| \times\left(\left|V a_{i}\right| \times\left|V a_{j}\right|\right),
$$

where

$$
a_{p 1}=\max \left\{a_{k 1}, a_{m 1}\right\}, a_{p 2}=\max \left\{a_{k 1}, a_{m 2}\right\}, \ldots, a_{p\left(\left|V a_{i}\right| \times\left|V a_{j}\right|\right)}=\max \left\{a_{k\left|V a_{i}\right|}, a_{m\left|V a_{j}\right|}\right\} .
$$

\subsection{Reduction Method}

A reduct is a minimal set of attributes that preserve the classification. In [17], we presented the application of multi-soft sets for finding reducts. The proposed approach is based on AND operation in multi-soft sets [16].

Definition 7. Let $(F, A)=\left(\left(F, a_{i}\right): 1 \leq i \leq|A|\right)$ be multi-soft set over $U$ representing a multi-valued information system $S=(U, A, V, f)$. A set of attributes $B \subseteq A$ is called a reduct for $A$ if

where

$$
\left.\left.C_{F\left(b_{1} \times \cdots \times b_{|B|}\right)}\right)=C_{F\left(a_{1} \times \cdots \times a_{|A|}\right)}\right) \text { and } C_{F\left(b_{1} \times \cdots \times b_{\left|B^{*}\right|}\right)} \neq C_{F\left(a_{1} \times \cdots \times a_{|A|}\right)}, \quad B^{*} \subset B,
$$

$$
\left(F, a_{i} \times a_{j}\right)=\left(F, a_{i}\right) \mathrm{AND}\left(F, a_{j}\right) .
$$

In [17], we explore the applicability of matrices representation for finding reducts. We show that the reducts obtained are equivalent with that in [20].

Proposition 2. Let $\mathcal{M}_{A}$ be a matrices over $U$ representing multi-soft sets $(F, A)$. A set of attributes $B \subseteq A$ is a reduct for $A$ if only if $C_{A_{N D M_{b}}: b \in B}=C_{A_{N D M_{a}}: a \in B}$. 
Proof. It is clear, since every $\left(F, a_{i}\right)$ can be represented as $M_{a_{i}}, 1 \leq i \leq|A|$ and vice versa.

Example 2. Let given a small dataset as follow.

Table 3. An Information System

\begin{tabular}{ccccl}
\hline$U / A$ & $a_{1}$ & \multicolumn{1}{c}{$a_{2}$} & \multicolumn{1}{c}{$a_{3}$} & \multicolumn{1}{c}{$a_{4}$} \\
\hline 1 & low & bad & Loss & small \\
2 & low & good & Loss & large \\
3 & high & good & Loss & medium \\
4 & high & good & Loss & medium \\
5 & low & good & Profit & large \\
\hline
\end{tabular}

Let $A=\left\{a_{1}, a_{2}, a_{3}, \overline{a_{4}}\right\}$, the multi-soft sets representing Table 3 is given as $(F, A)=\left(\begin{array}{l}\{\{\text { low }=1,2,5\},\{\text { high }=3,4\}\},\{\{\text { bad }=1\}, \text {, good }=2,3,4,5\}\}, \\ \{\{\text { loss }=1,2,3,4\},\{\text { profit }=5\}\},\{\{\text { small }=1\},\{\text { large }=2,5\},\{\text { medium }=3,4\}\}\end{array}\right)$, and the matrices representing $(F, A)$ is $\mathcal{M}_{A}=\left\{M_{a_{1}}, M_{a_{2}}, M_{a_{3}}, M_{a_{4}}\right\}$, where

$$
M_{a_{1}}=\left[\begin{array}{ll}
1 & 0 \\
1 & 0 \\
0 & 1 \\
0 & 1 \\
1 & 0
\end{array}\right], M_{a_{2}}=\left[\begin{array}{ll}
1 & 0 \\
0 & 1 \\
0 & 1 \\
0 & 1 \\
0 & 1
\end{array}\right], M_{a_{3}}=\left[\begin{array}{ll}
1 & 0 \\
1 & 0 \\
1 & 0 \\
1 & 0 \\
0 & 1
\end{array}\right] \text { and } M_{a_{4}}=\left[\begin{array}{lll}
1 & 0 & 0 \\
0 & 1 & 0 \\
0 & 0 & 1 \\
0 & 0 & 1 \\
0 & 1 & 0
\end{array}\right] \text {. }
$$

Since $A=\left\{a_{1}, a_{2}, a_{3}, a_{4}\right\}$, then

$$
M_{A N D a_{i}: 1 \leq i \leq 4}=\left[\begin{array}{llllllllllllllllllllllll}
1 & 0 & 0 & 0 & 0 & 0 & 0 & 0 & 0 & 0 & 0 & 0 & 0 & 0 & 0 & 0 & 0 & 0 & 0 & 0 & 0 & 0 & 0 & 0 \\
0 & 0 & 0 & 0 & 0 & 0 & 0 & 1 & 0 & 0 & 0 & 0 & 0 & 0 & 0 & 0 & 0 & 0 & 0 & 0 & 0 & 0 & 0 & 0 \\
0 & 0 & 0 & 0 & 0 & 0 & 0 & 0 & 0 & 0 & 0 & 0 & 0 & 0 & 0 & 0 & 0 & 0 & 0 & 0 & 1 & 0 & 0 & 0 \\
0 & 0 & 0 & 0 & 0 & 0 & 0 & 0 & 0 & 0 & 0 & 0 & 0 & 0 & 0 & 0 & 0 & 0 & 0 & 0 & 1 & 0 & 0 & 0 \\
0 & 0 & 0 & 0 & 0 & 0 & 0 & 0 & 0 & 0 & 1 & 0 & 0 & 0 & 0 & 0 & 0 & 0 & 0 & 0 & 0 & 0 & 0 & 0
\end{array}\right]
$$

where

$$
C_{M_{A N D a_{i}: 1 \leq i \leq 4}}=\{\{1\},\{2\},\{3,4\},\{5\}\} .
$$

Let, given $B=\left\{a_{1}, a_{2}, a_{3}\right\}$ and $C=\left\{a_{3}, a_{4}\right\}$, then we have

$$
M_{a_{1}} \text { ANDM }_{a_{2}} \text { ANDM }_{a_{3}}=\left[\begin{array}{cccccccc}
1 & 0 & 0 & 0 & 0 & 0 & 0 & 0 \\
0 & 0 & 1 & 0 & 0 & 0 & 0 & 0 \\
0 & 0 & 0 & 0 & 0 & 0 & 1 & 0 \\
0 & 0 & 0 & 0 & 0 & 0 & 1 & 0 \\
0 & 0 & 0 & 1 & 0 & 0 & 0 & 0
\end{array}\right]
$$

and

$$
M_{a_{3}} \mathrm{ANDM}_{a_{4}}=\left[\begin{array}{cccccc}
1 & 0 & 0 & 0 & 0 & 0 \\
0 & 1 & 0 & 0 & 0 & 0 \\
0 & 0 & 1 & 0 & 0 & 0 \\
0 & 0 & 1 & 0 & 0 & 0 \\
0 & 0 & 0 & 0 & 1 & 0
\end{array}\right]
$$

where

$C_{M_{a_{1}} \mathrm{AND} M_{a_{2}} \mathrm{ANDM} a_{3}}=\{\{1\},\{2\},\{3,4\},\{5\}\} \quad$ and $C_{M_{a_{1}} \mathrm{AND} M_{a_{2}} \mathrm{AND} M_{a_{3}}}=\{\{1\},\{2\},\{3,4\},\{5\}\}$, respectively. Thus, we have $\left\{a_{1}, a_{2}, a_{3}\right\}$ and $\left\{a_{3}, a_{4}\right\}$ are reducts of $A$. Furthermore, core is

$$
\left\{a_{1}, a_{2}, a_{3}\right\} \cap\left\{a_{3}, a_{4}\right\}=\left\{a_{3}\right\}
$$


Thus a reduct is a set of attributes that preserves classes. It means that a reduct is the minimal subset of attributes that enables the same classification of elements of the universe as the whole set of attributes. In other words, attributes that do not belong to a reduct are superfluous with regard to classification of elements of the universe.

\subsection{Dataset}

The second part of this article starts from a description of the traditional Malay musical instrument sounds dataset and the feature selection problem for which the data was collected. This work will identify which attributes are dispensable and further can be removed from dataset without affected the original classification power. Then, we make a decision of the reduced dataset based on the attributes dispensed. From this experiment, the ideal dataset can be represented in decision system form as $S=\{U, A \cup\{d\}, V, f\}$. There are 1116 instances in the universe $U$, with 37 attributes of $A$ as the set of condition attributes (as shown in Table 4) and the family of the instruments as the decision attribute $d$. The distribution of all instances in each class is uniform with no missing values in the data [21].

Table 4. Features Descriptions

\begin{tabular}{|c|l|}
\hline Number & \multicolumn{1}{|c|}{ Description } \\
\hline 1 & Zero Crossing \\
\hline $2-3$ & Mean and Standard Deviation of Zero Crossings Rate \\
\hline $4-5$ & Mean and Standard Deviation of Root-Mean-Square \\
\hline $6-7$ & Mean and Standard Deviation of Spectral Centroid \\
\hline $8-9$ & Mean and Standard Deviation of Bandwidth \\
\hline $10-11$ & Mean and Standard Deviation of Flux \\
\hline $12-37$ & Mean and Standard Deviation of the First 13 MFCCs \\
\hline
\end{tabular}

The mean and standard deviation were then calculated for each of these features. The problem of feature selection trough the above dataset is how to delete irrelevant attributes. It can be done, among all attributes we want to remove dispensable attributes. To select the candidate of the dispensable attributes, we employ the method proposed by [17].

\subsection{Result and Discussion}

The decision table used in this study is in the form of continuous value with noncategorical features (attributes). In order to employ the proposed method, it is essential to transform the dataset into categorical ones. For that, the equal width binning in [18] is used and the dataset is first discretized into 3 categorical values as shown in Table 5.

Table 5. Summary of Data Distribution

\begin{tabular}{|l|l|c|c|c|}
\hline \multicolumn{1}{|c|}{ Attribute } & Description & $\mathbf{\# 1}$ & $\mathbf{\# 2}$ & $\mathbf{\# 3}$ \\
\hline ZC & Zero Crossing & 456 & 438 & 222 \\
\hline MEANZCR & Mean of Zero Crossings Rate & 134 & 638 & 344 \\
\hline STDZCR & Standard Deviation of Zero Crossings Rate & 1092 & 16 & 8 \\
\hline MEANRMS & Mean of Root-Mean-Square & 724 & 386 & 6 \\
\hline STDRMS & Standard Deviation of Root-Mean-Square & 350 & 498 & 268 \\
\hline MEANC & Mean of Spectral Centroid & 198 & 528 & 390 \\
\hline STDC & Standard Deviation of Spectral Centroid & 166 & 430 & 520 \\
\hline MEANB & Mean of Bandwidth & 206 & 336 & 574 \\
\hline STDB & Standard Deviation of Bandwidth & 480 & 474 & 162 \\
\hline MEANFLUX & Mean of Flux & 484 & 494 & 138 \\
\hline
\end{tabular}




\begin{tabular}{|l|l|c|c|c|}
\hline STDFLUX & Standard Deviation of Flux & 692 & 304 & 120 \\
\hline MMFCC1 & Mean of the MFCCs \#1 & 1116 & 0 & 0 \\
\hline MMFCC2 & Mean of the MFCCs \#2 & 984 & 96 & 36 \\
\hline MMFCC3 & Mean of the MFCCs \#3 & 302 & 516 & 298 \\
\hline MMFCC4 & Mean of the MFCCs \#4 & 692 & 352 & 72 \\
\hline MMFCC5 & Mean of the MFCCs \#5 & 596 & 392 & 128 \\
\hline MMFCC6 & Mean of the MFCCs \#6 & 822 & 246 & 48 \\
\hline MMFCC7 & Mean of the MFCCs \#7 & 840 & 222 & 54 \\
\hline MMFCC8 & Mean of the MFCCs \#8 & 924 & 142 & 50 \\
\hline MMFCC9 & Mean of the MFCCs \#9 & 730 & 380 & 6 \\
\hline MMFCC10 & Mean of the MFCCs \#10 & 938 & 136 & 42 \\
\hline MMFCC11 & Mean of the MFCCs \#11 & 950 & 122 & 44 \\
\hline MMFCC12 & Mean of the MFCCs \#12 & 950 & 144 & 22 \\
\hline MMFCC13 & Mean of the MFCCs \#13 & 1010 & 42 & 64 \\
\hline SMFCC1 & Standard Deviation of the MFCCs \#1 & 1116 & 0 & 0 \\
\hline SMFCC2 & Standard Deviation of the MFCCs \#2 & 856 & 218 & 42 \\
\hline SMFCC3 & Standard Deviation of the MFCCs \#3 & 772 & 196 & 148 \\
\hline SMFCC4 & Standard Deviation of the MFCCs \#4 & 778 & 256 & 82 \\
\hline SMFCC5 & Standard Deviation of the MFCCs \#5 & 724 & 264 & 128 \\
\hline SMFCC6 & Standard Deviation of the MFCCs \#6 & 786 & 176 & 154 \\
\hline SMFCC7 & Standard Deviation of the MFCCs \#7 & 846 & 172 & 98 \\
\hline SMFCC8 & Standard Deviation of the MFCCs \#8 & 864 & 162 & 90 \\
\hline SMFCC9 & Standard Deviation of the MFCCs \#9 & 808 & 220 & 88 \\
\hline SMFCC10 & Standard Deviation of the MFCCs \#10 & 752 & 292 & 72 \\
\hline SMFCC11 & Standard Deviation of the MFCCs \#11 & 898 & 156 & 62 \\
\hline SMFCC12 & Standard Deviation of the MFCCs \#12 & 986 & 98 & 32 \\
\hline MFCC13 & Standard Deviation of the MFCCs \#13 & 840 & 148 & 128 \\
\hline & & & & \\
\hline
\end{tabular}

To find the reducts (relevant features), the proposed model is implemented in MATLAB version 7.6.0.324 (R2008a). It is executed on a processor Intel Core 2 Duo CPUs. The total main memory is 1 gigabyte and the operating system is Windows XP Professional SP3. The results of the selected features are presented in Table 6 as follow:

Table 6. The Selected Attributes

\begin{tabular}{|l|l|}
\hline \multicolumn{1}{|c|}{ Attribute } & \multicolumn{1}{c|}{ Description } \\
\hline ZC & Zero Crossing \\
\hline MEANZCR & Mean of Zero Crossings Rate \\
\hline STDZCR & Standard Deviation of Zero Crossings Rate \\
\hline MEANRMS & Mean of Root-Mean-Square \\
\hline STDRMS & Standard Deviation of Root-Mean-Square \\
\hline MEANC & Mean of Spectral Centroid \\
\hline STDC & Standard Deviation of Spectral Centroid \\
\hline MEANB & Mean of Bandwidth \\
\hline STDB & Standard Deviation of Bandwidth \\
\hline MEANFLUX & Mean of Flux \\
\hline STDFLUX & Standard Deviation of Flux \\
\hline MMFCC1 & Mean of the MFCCs \#1 \\
\hline MMFCC2 & Mean of the MFCCs \#2 \\
\hline MMFCC3 & Mean of the MFCCs \#3 \\
\hline MMFCC4 & Mean of the MFCCs \#4 \\
\hline MMFCC5 & Mean of the MFCCs \#5 \\
\hline
\end{tabular}




\begin{tabular}{|l|l|}
\hline MMFCC6 & Mean of the MFCCs \#6 \\
\hline MMFCC7 & Mean of the MFCCs \#7 \\
\hline MMFCC8 & Mean of the MFCCs \#8 \\
\hline MMFCC9 & Mean of the MFCCs \#9 \\
\hline MMFCC10 & Mean of the MFCCs \#10 \\
\hline MMFCC11 & Mean of the MFCCs \#11 \\
\hline MMFCC12 & Mean of the MFCCs \#12 \\
\hline MMFCC13 & Mean of the MFCCs \#13 \\
\hline SMFCC1 & Standard Deviation of the MFCCs \#1 \\
\hline SMFCC2 & Standard Deviation of the MFCCs \#2 \\
\hline SMFCC3 & Standard Deviation of the MFCCs \#3 \\
\hline SMFCC4 & Standard Deviation of the MFCCs \#4 \\
\hline SMFCC5 & Standard Deviation of the MFCCs \#5 \\
\hline SMFCC6 & Standard Deviation of the MFCCs \#6 \\
\hline SMFCC7 & Standard Deviation of the MFCCs \#7 \\
\hline SMFCC8 & Standard Deviation of the MFCCs \#8 \\
\hline SMFCC9 & Standard Deviation of the MFCCs \#9 \\
\hline SMFCC10 & Standard Deviation of the MFCCs \#10 \\
\hline SMFCC11 & Standard Deviation of the MFCCs \#11 \\
\hline SMFCC12 & Standard Deviation of the MFCCs \#12 \\
\hline MFCC13 & Standard Deviation of the MFCCs \#13 \\
\hline
\end{tabular}

From Table 6, the result shows that the proposed model successfully generates the best features set with 35 attributes from 37 of original full attributes. It is found that $\{$ MMFCC1, SMFCC1 $\}$ is the dispensable set of attributes. Thus, the relevant features (reduct) is $\mathrm{A}-\{$ MMFCC1, SMFCC1 .

\section{Conclusion}

In this paper, the matrices computation of multi-soft sets has been used as feature selection (attribute reduction) technique to identify the best features of Traditional Malay musical instrument sounds. To perform this task, two categories of features schemes which are perception-based and MFCC which consist of 37 attributes are extracted. The equal width and equal frequency binning discretization technique is then employed to transform this continuous-value with non-categorical features (attributes) into categorical form. Afterward, the multi-soft sets approach is adopted for feature selection through dimensionality reduction in multi-valued domain. It is found that 35 best features are successfully generated by the proposed model. For that, our future work will examine others feature selection technique and examine the effectiveness of the selected features towards the classification performance.

\section{Acknowledgement}

This work is supported by UPI-YPTK Research Grant ref number 065/UPIYPTK/RG/II/2014.

\section{References}

[1] D. Molodtsov, "Soft Set Theory-First Results", Computer and Mathematics with Applications, vol. 37, (1999), pp. 19-31.

[2] M. Xiuqin, N. Sulaiman, Q. Hongwu and T. Herawan, "A Novel Normal Parameter Reduction Algorithm of Soft Sets", In Gaspar Cunha et al. (Eds.): AISC 2011, Advances in Intelligent and Soft Computing, vol. 96, (2011), pp. 221-229. 
[3] A. N. M. Rose, H. Hassan, M. I. Awang, T. Herawan and M. M. Deris, "Solving Incomplete Datasets in Soft Set Using Parity Bits of Supported Sets", In T. H. Kim, et al., (Eds.): UCMA 2011, Communication of Computer and Information Sciences, vol. 151, (2011), pp. 33-43.

[4] M. Xiuqin, N. Sulaiman, Q. Hongwu, J. M. Zain and T. Herawan, "A New Efficient Normal Parameter Reduction Algorithm of Soft Sets", Computer and Mathematics with Applications, vol. 62, no. 2, (2011), pp. 588-598.

[5] T. Herawan and M. M. Deris, "A soft set approach for association rules mining", Knowledge Based Systems, vol. 24, no. 1, (2011), pp. 186-195.

[6] A. N. M. Rose, M. I. Awang, T. Herawan and M. M. Deris, "Hybrid Reduction in Soft Set Decision Making", In De-Shuang Huang, et al., (Eds.): ICIC 2011, Lecture Notes in Computer Sciences, vol. 6838, (2011), pp. 108-115.

[7] Q. Hongwu, M. Xiuqin, T. Herawan and J. M. Zain, "Data filling approach of soft sets under incomplete information”, In N. T. Nguyen, C. G. Kim, A. Janiak (Eds.): ACIIDS 2011, Lecture Notes in Computer Science, vol. 6592, (2011), pp. 302-311.

[8] Q. Hongwu, M. Xiuqin, T. Herawan and J. M. Zain, "An adjustable approach to interval-valued intuitionistic fuzzy soft sets based decision making", In N. T. Nguyen, C. G. Kim, A. Janiak (Eds.): ACIIDS 2011, Lecture Notes in Computer Science, vol. 6592, (2011), pp. 80-89.

[9] R. Mamat, T. Herawan and M. M. Deris, "Super Attribute Representative for Decision Attribute Selection", In J. M. Zain, et al., (Eds.): ICSECS 2011, Communication of Computer and Information Sciences, vol. 188, no. II, (2011), pp. 516-529.

[10] Q. Hongwu, M. Xiuqin, J. M. Zain, N. Sulaiman and T. Herawan, "A Soft Set Model on Information System and Its Application in Clustering Attribute Selection", In J. M. Zain, et al., (Eds.): ICSECS 2011, Communication of Computer and Information Sciences, vol. 188, no. II, (2011), pp. 16-27.

[11] M. I. Awang, A. N. M. Rose, T. Herawan and M. M. Deris, "Soft set approach for selecting a clustering attribute", In L. Chao, J. Zhong and Y. Feng (Eds.): ADMA 2010, Lecture Notes in Computer Science, vol. 6441, (2010), pp. 87-98.

[12] A. N. M. Rose, T. Herawan and M. M. Deris, "A framework of decision making based on maximal supported sets", In L. Zhang, J. Kwok and B.-L. Lu (Eds.): ISNN 2010, Part I, Lecture Notes in Computer Science, vol. 6063, (2010), pp. 473-482.

[13] T. Herawan and M. M. Deris, "Soft decision making for patients suspected influenza", In D. Taniar, et al., (Eds.): ICCSA 2010, Part III, Lecture Notes in Computer Science, vol. 6018, (2010), pp. 405-418.

[14] T. Herawan, A. N. M. Rose and M. D. Mustafa, "Soft set theoretic approach for dimensionality reduction”, In D. Ślęzak et al. (Eds.): DTA 2009, Communication of Computer and Information Sciences, vol. 64, (2009), pp. 180-187.

[15] T. Herawan, I. T. R. Yanto and M. M. Deris, "Soft set approach for maximal association rules mining, In D. Ślęzak, et al., (Eds.): DTA 2009, Communication of Computer and Information Sciences, vol. 64, (2009), pp. 163-170.

[16] T. Herawan and M. D. Mustafa, "On multi-soft sets construction in information systems", In D. S. Huang, et al., (Eds.): ICIC 2009, Lecture Notes in Artificial Intelligence, vol. 5755, (2009), pp. 101-110.

[17] T. Herawan, M. D. Mustafa and J. H. Abawajy, "Matrices representation of multi soft-sets and its application”, In D. Taniar et al. (Eds.): ICCSA 2010, Part III, Lecture Notes in Computer Science, vol. 6018, (2010), pp. 201-214.

[18] S. Palaniappan and T. K. Hong, "Discretization of Continuous Valued Dimensions in OLAP Data Cubes", IJCSNS, International Journal of Computer Science and Network Security, vol. 8, (2008), pp. $116-126$.

[19] P. K. Maji and A. R. Roy, "An application of soft sets in a decision making problem”, Computers and Mathematics with Applications, vol. 44, (2002), pp. 1077-1083.

[20]Z. Pawlak and A. Skowron, "Rudiments of rough sets", Information Sciences, vol. 177, no. 1, (2007), pp. 3-27.

[21] N. Senan, R. Ibrahim, N. M. Nawi, M. M. Mokji and T. Herawan, "The Ideal Data Representation for Feature Extraction of Traditional Malay Musical Instrument Sounds Classification”, In De-Shuang Huang, et al., (Eds.): ICIC 2010, Lecture Notes in Computer Science, vol. 6215, (2010), pp. 345-353. 